Marquette University

e-Publications@Marquette

Biomedical Engineering Faculty Research and

Publications

Biomedical Engineering, Department of

3-2005

\title{
Antegrade Iliac Artery Stent Implantation for the Temporal and Spatial Examination of Stent-Induced Neointimal Hyperplasia and Alterations in Regional Fluid Dynamics
}

\author{
John F. LaDisa \\ Marquette University, john.ladisa@marquette.edu \\ Heidi T. Meier \\ Clement J. Zablocki Veterans Affairs Medical Center \\ Lars E. Olson \\ Marquette University, lars.olson@marquette.edu \\ Judy R. Kersten \\ Medical College of Wisconsin \\ David C. Warltier \\ Marquette University
}

See next page for additional authors

Follow this and additional works at: https://epublications.marquette.edu/bioengin_fac

Part of the Biomedical Engineering and Bioengineering Commons

\section{Recommended Citation}

LaDisa, John F.; Meier, Heidi T.; Olson, Lars E.; Kersten, Judy R.; Warltier, David C.; and Pagel, Paul S., "Antegrade Iliac Artery Stent Implantation for the Temporal and Spatial Examination of Stent-Induced Neointimal Hyperplasia and Alterations in Regional Fluid Dynamics" (2005). Biomedical Engineering Faculty Research and Publications. 215.

https://epublications.marquette.edu/bioengin_fac/215 


\section{Authors}

John F. LaDisa, Heidi T. Meier, Lars E. Olson, Judy R. Kersten, David C. Warltier, and Paul S. Pagel

This article is available at e-Publications@Marquette: https://epublications.marquette.edu/bioengin_fac/215 
Marquette University

e-Publications@Marquette

\section{Biomedical Engineering Faculty Research and Publications/College of} Engineering

This paper is NOT THE PUBLISHED VERSION; but the author's final, peer-reviewed manuscript. The published version may be accessed by following the link in the citation below.

Journal of Pharmacological and Toxicological Methods, Vol. 51, No. 2 (March/April 2005): 115-121. DOI. This article is (C) Elsevier and permission has been granted for this version to appear in $\underline{\mathrm{e}-}$ Publications@Marquette. Elsevier does not grant permission for this article to be further copied/distributed or hosted elsewhere without the express permission from Elsevier.

\section{Antegrade Iliac Artery Stent Implantation for The Temporal and Spatial Examination of Stent-Induced Neointimal Hyperplasia and Alterations in Regional Fluid Dynamics}

John F. LaDisa Jr.

Department of Anesthesiology, Medical College of Wisconsin, Milwaukee, WI Clement J. Zablocki Veterans Affairs Medical Center, Milwaukee, WI, United States Department of Biomedical Engineering, Marquette University, Milwaukee, WI Heidi T. Meier Clement J. Zablocki Veterans Affairs Medical Center, Milwaukee, WI Lars E. Olson Clement J. Zablocki Veterans Affairs Medical Center, Milwaukee, WI Department of Biomedical Engineering, Marquette University, Milwaukee, WI Judy R .Kersten 
Department of Anesthesiology, Medical College of Wisconsin, Milwaukee, WI Department of Pharmacology and Toxicology, Medical College of Wisconsin, Milwaukee, WI

\title{
David C. Warltier
}

Department of Anesthesiology, Medical College of Wisconsin, Milwaukee, WI

Department of Biomedical Engineering, Marquette University, Milwaukee, WI

Division of Cardiovascular Diseases, Department of Medicine, Medical College of Wisconsin, Milwaukee, WI

\section{Paul S. Pagel}

Department of Anesthesiology, Medical College of Wisconsin, Milwaukee, WI, USA

Clement J. Zablocki Veterans Affairs Medical Center, Milwaukee, WI, United States

Department of Biomedical Engineering, Marquette University, United States

\begin{abstract}
Introduction; Neointimal hyperplasia remains an important problem after stent implantation. Previous investigations examining vascular responses to stent implantation and effects of drugs have used a retrograde deployment approach that may inadvertently alter the local fluid dynamics surrounding the stent. We present a model of antegrade iliac artery stent implantation that facilitates the analysis of stent-induced alterations in neointimal hyperplasia and wall shear stress in vivo.Methods: Stent delivery catheters were inserted through the left carotid artery in anesthetized rabbits $(n=37)$. Catheters were advanced under fluoroscopic guidance to the distal iliac arteries, where the stent was deployed. Hemotoxylin and eosin (H\&E) staining of unstented and stented vascular sections was performed 21 days after implantation. Results: Selective unilateral stent implantation was successful in 32 of 37 rabbits. No histological abnormalities were observed in the aorta, contralateral unstented iliac, or distal femoral arteries. Neointimal hyperplasia was localized to the stented region.Discussion: The model of stent implantation was relatively easy to perform and produced selective neointimal hyperplasia within the stented region without evidence of damage, cellular proliferation, or flow disruption in the surrounding normal arterial vessels. The model will allow detailed examination of the influence of stent implantation on indices of wall shear stress, neointimal hyperplasia, the mechanisms of cellular proliferation in vivo, and their modification by drugs.
\end{abstract}

\section{Keywords}

Iliac artery, Stent implantation, Hyperplasia

\section{Introduction}

Restenosis after stent implantation remains an important clinical problem (Fischman et al., 1994, Holmes et al., 1998, Serruys et al., 1994, van Beusekom et al., 1998). Stent implantation acutely restores blood flow distal to vascular stenoses but may be associated with cellular damage (Rogers et al., 1999, Schwartz et al., 1992). Recent data indicate that spatial distributions of shear stress mediate the rate and location of smooth muscle cell migration and proliferation after vascular injury (Liu \& Goldman, 2001, Liu et al., 2002), suggesting that stent geometry and deployment ratio may differentially influence restenosis after implantation. We have previously demonstrated that design properties unique to the geometry of an implanted stent differentially influence the area of idealized computational vessels subjected to indices of wall shear stress associated with vascular susceptibility to neointimal hyperplasia using 3D computational fluid dynamics models (LaDisa et al., 2003, LaDisa et al., 2004). The current report presents a methodology that has been developed to test these computational findings experimentally using an antegrade stent implantation model in rabbits. 
Previous models of stent implantation in rabbit iliac arteries have used retrograde stent placement performed through the femoral artery (Burgio et al., 1984, Garasic et al., 2000, Herdeg et al., 2003). However, this retrograde model may introduce alterations in the localized flow environment distal to the stent, which may subsequently influence cellular proliferation within the stented region, and is not representative of typical catheter entrance to regional vascular lesions. We present an antegrade model of stent implantation that can be used to investigate spatial and temporal alterations in neointimal hyperplasia within the interventional region in the absence of flow disturbances introduced by the method of deployment. We have preliminarily reported the use of this model to compare temporal alterations in vascular histology with concomitant alterations in indices of wall shear stress after stent implantation (LaDisa, Warltier et al., 2004). These data suggest a high correlation between areas of low wall shear stress and neointimal hyperplasia within the stented zone. We also demonstrate how the model can also be employed to analyze the local expression of molecular mediators that contribute to cellular proliferation within the stented region to elucidate mechanisms of restenosis.

\section{Methods}

\subsection{Use and care of animals}

All experimental procedures and protocols used in this investigation were approved by the Animal Care and Use Committee of Marquette University and the Medical College of Wisconsin. Furthermore, all conformed to the Guiding Principles in the Care and Use of Animals of the American Physiological Society and the Guide for the Care and Use of Laboratory Animals as outlined by the National Institutes of Health (7th ed. Washington, DC: Nat. Acad. Press, 1996).

\subsection{Antegrade iliac artery stent implantation}

Male New Zealand white rabbits $(n=37)$ weighing approximately $3.5 \mathrm{~kg}$ were obtained from New Franken Researchers (New Franken, WI). The rabbit iliac artery was selected for stent implantation because the diameter and length of this vessel closely approximates the size of stents frequently used in clinical settings (3.0 and 16 $\mathrm{mm}$, respectively). The absence of axial curvature in these arteries and the ability to obtain experimental (stent) and chronic control data in the contralateral iliac arteries of a single animal are also important benefits of this model. Stent implantation was performed under sterile conditions using portable fluoroscopic imaging (OEC, GE Medical Systems, Milwaukee, WI). Rabbits were anesthetized intravenously through the right marginal ear vein with a mixture of xylazine $(2.2 \mathrm{mg} / \mathrm{kg})$ and ketamine $(22 \mathrm{mg} / \mathrm{kg})$ and intubated using a 3.0-mm pediatric endotracheal tube. Anesthesia was maintained with $1 \%$ to $2 \%$ isoflurane in oxygen, and rabbits were allowed to breathe spontaneously. Body temperature was controlled at $37^{\circ} \mathrm{C}$ through the use of a heating pad.

An incision was made above the left carotid artery and the vessel dissected free. A $5 F$ introducer sheath (Avanti+, Cordis, Miami, FL) was inserted into the vessel, through which a fluid-filled 5F JR3.5-5 softip guide catheter, preloaded with a guide wire (Entre II; Boston Scientific, Maple Grove, MN), was advanced from the carotid artery into the distal descending aorta. The guide wire was then advanced to the proximal portion of the right iliac artery. The contralateral iliac artery was not instrumented and served as a control. The guide catheter was carefully removed while the wire remained in place. The stent delivery system was advanced to the distal portion of the iliac artery using the guide wire and the stent was deployed to a pressure of 5 atm to achieve a stent-to-artery-size ratio range of 1.1 to 1.2:1 (Garasic et al., 2000). Intravenous heparin (100 U/kg) was administered immediately after stent deployment (Rogers \& Edelman, 1995). Successful deployment was verified by angiography, and the stent delivery catheter was removed. The left carotid artery was ligated, the incision above the carotid artery closed in layers, and the rabbit allowed to emerge from anesthesia.

Intramuscular buprenorphine $(0.05 \mathrm{mg} / \mathrm{kg}, \mathrm{BID})$ was used for the treatment of postoperative pain. Rabbits also received antibiotic prophylaxis (enrofloxacin, $5 \mathrm{mg} / \mathrm{kg}$ ) for 4 days after stent implantation and aspirin in their drinking water ( $20 \mathrm{mg} /$ day) for the duration of the protocol (Rogers \& Edelman, 1995). 


\subsection{Histological analysis}

Additional rabbits $(n=12)$ were used for histological analysis of stented and unstented arteries. Rabbits underwent iliac artery stent implantation as described above and were sacrificed 21 days after deployment. On the day of sacrifice, rabbits were anesthetized as previously described, the chest opened by a midline sternotomy, and the pericardium incised. The apex of the left ventricle was perfused at a mean arterial pressure of $100 \mathrm{~mm} \mathrm{Hg}$ with lactated Ringer's solution, followed by Carnoy's solution (Rogers, Edelman, \& Simon, 1998) while the rabbit was simultaneously exsanguinated through a right atrial incision. The proximal and distal ends of the iliac arteries were identified, and the vessels were carefully excised and fixed in Carnoy's solution for $4 \mathrm{~h}$. Samples were then subjected to iterative dehydration and embedded in glycol methyl methacrylate (Technovit 7100, EB Sciences, Agawam, MA).

Specimens were sectioned at room temperature in 5- $\mu \mathrm{m}$ intervals using a microtome equipped with a tungsten carbide knife that facilitated direct sectioning through the stainless steel linkages of the implanted stent (Delaware Diamond Knives, Ted Pella, Redding, CA). Histological sections were floated on a warm water bath, mounted on positively charged Colorfrost Plus microscope slides (Fisher Scientific, Pittsburgh, PA), dried, and refrigerated at $4{ }^{\circ} \mathrm{C}$ until histological analysis. Hemotoxylin and eosin (H\&E) staining was used to identify the nuclei, cytoplasmic structures, and extracellular components in stented and unstented arterial sections. Light photomicrographs were obtained using a Nikon Optiphot-2 microscope (Tokyo, Japan) equipped with a Diagnostics SPOT CCD camera (Sterling Heights, MI).

\subsection{Immunohistochemistry}

Sections were treated with $0.1 \%$ trypsin in phosphate-buffered saline (PBS) at room temperature for $1 \mathrm{~h}$ prior to staining to increase the permeability of the plastic sections (Burgio et al., 1984). Sections were then incubated with a 1:200 dilution of a mouse monoclonal primary antibody for $\alpha$-smooth muscle actin (Sigma, St. Louis, MO) in PBS at $37^{\circ} \mathrm{C}$ for $30 \mathrm{~min}$. Sections were washed three times for 5 min with PBS and subsequently incubated with a 1:200 dilution of biotinylated antimouse secondary antibody (Amersham Pharmacia Biotech, UK) in PBS at $37^{\circ} \mathrm{C}$ for $30 \mathrm{~min}$. Sections were washed three times for $5 \mathrm{~min}$ with PBS before conjugation with $10 \mu \mathrm{g} / \mathrm{ml}$ streptavidin-labeled Texas Red (sulfonyl chloride; Pierce, Rockford, IL) at $37^{\circ} \mathrm{C}$ for $15 \mathrm{~min}$. Sections were again washed three times for 5 min with PBS, and nuclear staining was achieved by including $1 \mu \mathrm{m}$ of TO-PRO-3 (Molecular Probes, Eugene, OR) during the final rinse. Images were obtained using a laser fluorescence imaging system and a confocal microscope. A krypton-argon laser was used for excitation wavelengths of 545 and 642 $\mathrm{nm}$, and emitted fluorescence was determined after long-pass filtering at corresponding wavelengths of 578 and $661 \mathrm{~nm}$ for Texas Red and TO-PRO, respectively.

\section{Results}

Thirty-seven rabbits were anesthetized, and 32 stents were successfully implanted. The average experimental time was $77 \pm 25 \mathrm{~min}$ (mean \pm S.D.) from induction until emergence from general anesthesia. The introduction of stent delivery catheters through the left carotid artery facilitated access to the distal iliac arteries where stent deployment was performed. The transition from the common carotid artery to the aortic arch presented an anatomical obstacle to stent implantation in four initial experiments. This difficulty was overcome in subsequent placement attempts by using contrast media injected into the aortic arch through JR3.5-5 softip guide catheters. The guide catheter was orientated dorsally under fluoroscopic guidance, and the preloaded guide wire was directed away from the aortic valve into the ascending aorta. The guide catheter was subsequently advanced, the guide wire retracted into the catheter, and the two carefully advanced into the distal aorta. The iliac artery illumination using contrast media, positioning of the stent delivery catheter, deployment and mechanical deformation of the associated stent, and removal of the delivery catheter are illustrated in Fig. 1. One additional 
rabbit was sacrificed because embolization of a thrombus into the distal iliac artery occurred immediately after stent implantation.
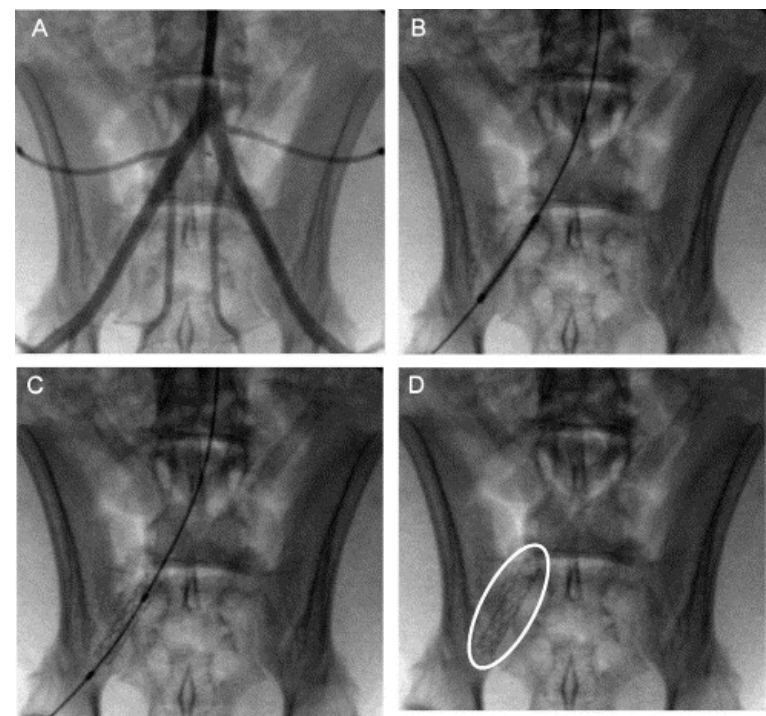

Fig. 1. Angiographic images of antegrade stent implantation. A 5F guide catheter and guide wire were introduced through the carotid artery and advanced into the distal descending aorta. A contrast media was administered to identify the iliac arteries (A). The guide wire was then advanced to the right iliac artery. A stent delivery system was advanced to the artery using the guide wire (B), and the stent was carefully deployed to 5 atm to achieve a stent-to-artery ratio range of 1.1 to 1.2:1 (C). The stent delivery catheter was then removed, leaving the implanted stent, as depicted within the ellipsoid of Panel D.

Antegrade stent implantation into the right iliac artery was not associated with histological evidence of aortic injury (Fig. 2A). Stent implantation also did not alter the histology of the contralateral iliac or distal femoral arteries, as indicated by the intact endothelial, internal elastic lamina, and medial layers (Fig. 2B to D). Histological analysis of stented iliac arteries revealed neointimal hyperplasia localized to the stented portion of the artery (Fig. 3). Immunohistochemical analysis of unstented vessels confirmed that that cells within the medial layer were of $\alpha$-actin smooth muscle origin (Fig. 4, left). Autofluorescence of the internal elastic lamina was detected and separated the boundary of the medial layer from the nuclei of endothelial cells lining the luminal surface. These cells and those within the medial and advential layers were successfully stained by TOPRO-3. The fluorescent photomicrograph presented in the right panel of Fig. 4 reveals stretching of the internal elastic lamina caused by stent placement, consistent with Fig. 3. The figure also demonstrates that much of the neointimal hyperplasia contains cells positive for $\alpha$-actin. Fig. 4 further illustrates that there are regions adjacent to the stent strut and lining the luminal surface that are devoid of smooth muscle $\alpha$-actin, but do contain intact nuclei. 


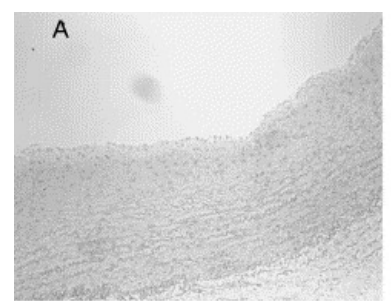

C
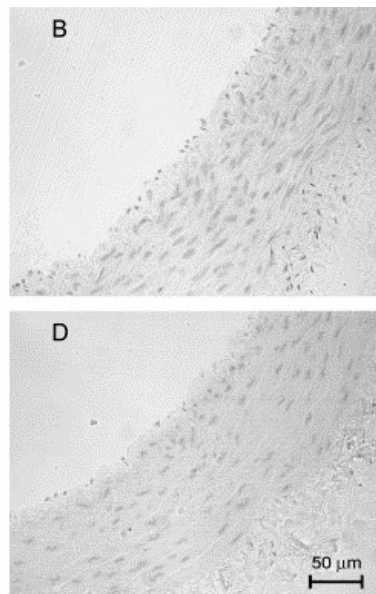

Fig. 2. Histological sections stained with H\&E illustrating normal intimal, medial, and adventitial layers of a descending aorta $(A, \times 10)$, contralateral unstented iliac artery $(B, \times 20)$, and left and right femoral arteries $(C$ and $D$, respectively, $\times 20)$ after antegrade stent implantation. Iliac artery cross-sections are arranged with the vascular lumen orientated toward the upper left corner of each image.

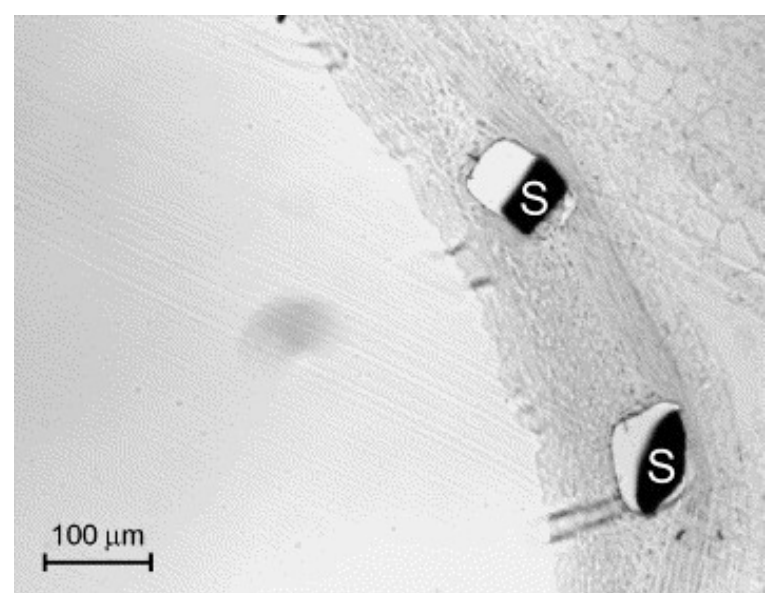

Fig. 3. Histological cross-section stained with H\&E within the stented portion of an interventional iliac artery $(\times 10)$. The iliac artery cross-section is arranged with the vascular lumen $(L)$ orientated toward the left side of the image, and stent struts (S) have been labeled for clarity.
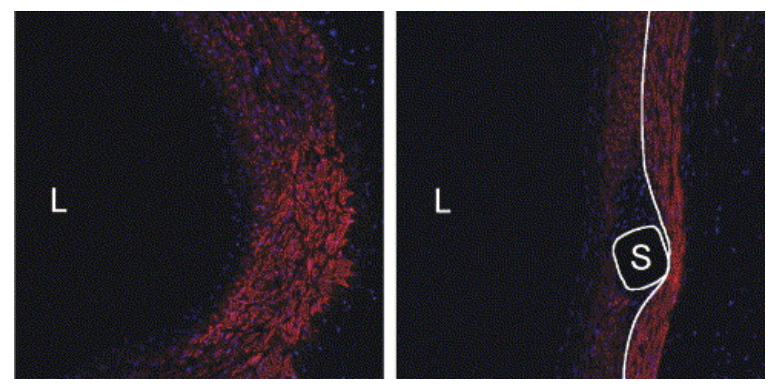

Fig. 4. Fluorescence photomicrographs $(\times 10)$ of contralateral unstented and stented iliac arteries stained with antibodies against $\alpha$-actin smooth muscle and cell nuclei. The iliac artery cross-sections are arranged with the vascular lumen $(L)$ orientated toward the left side of the image. The internal elastic lamina has been traced, and stent struts have been outlined and labeled for clarity. 


\section{Discussion}

We report a model of antegrade stent implantation that does not produce vascular injury and preserves the flow domain near the site of an implanted stent. Previous studies were conducted in rabbit iliac arteries using a retrograde stent implantation technique through the femoral artery (Garasic et al., 2000, Herdeg et al., 2003). These previous studies have provided information about the stent-induced development of neointimal hyperplasia, but neglected potentially influential alterations in the blood flow environment within the stented region after deployment. The current histological results of the stented region demonstrated appreciable neointimal growth within this vascular segment. Our preliminary data shown in Fig. 5 indicate that these histological findings correlate closely with temporal alterations in spatial distributions of wall shear stress, as determined from the computational fluid dynamics analysis of unstented and stented iliac artery flow domains obtained using microfocal X-ray computed tomography imaging (LaDisa, Warltier et al., 2004). The figure depicts normalized distributions of wall shear stress in an unstented iliac artery and stented iliac arteries immediately after stent deployment, or 21 days after implantation, using the methods presented in this report.

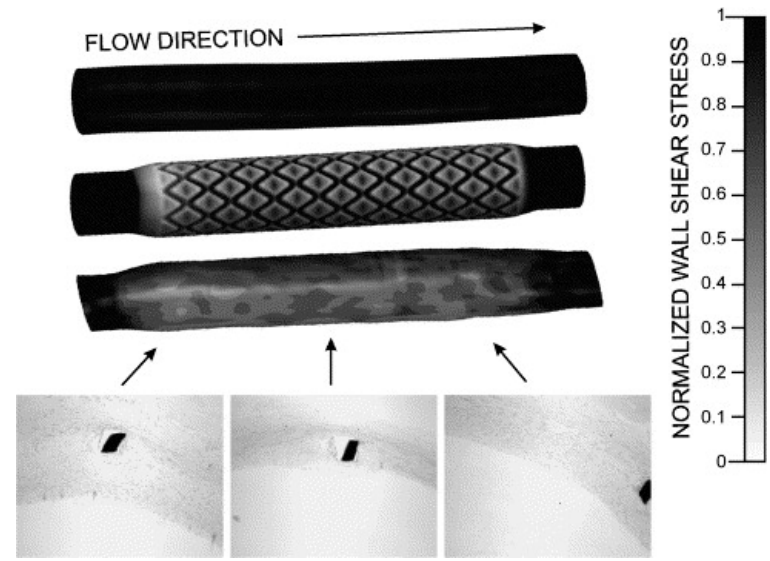

Fig. 5. Normalized distributions of wall shear stress in an unstented iliac artery (top) and stented iliac arteries immediately after deployment (theoretical, middle) or 21 days after antegrade stent implantation in vivo (bottom). Histological cross-section stained with H\&E $(\times 10)$ in the proximal, middle, and distal regions of the stent 21 days after implantation are depicted according to their respective spatial locations.

Previous studies have demonstrated that regions of low wall shear stress and elevated spatial wall shear stress gradients may be spatially colocalized to sites of neointimal hyperplasia (Kleinstreuer et al., 2001). Distributions of wall shear stress are lower within the stented region of the vessel, and this reduction in wall shear stress is most pronounced at the inlet of the stented region (Fig. 5). We have also recently reported that elevated spatial wall shear stress gradients are greatest in transition regions between the stent and native vessel as a result of the increased luminal diameter necessary to firmly anchor the stent into the vessel wall (LaDisa, Olson et al., 2004). Interestingly, analyses of histological cross-sections corresponding to the proximal, middle, and distal regions of the stent, 21 days after implantation, reveal appreciable neointimal hyperplasia at the entrance and exit of the stent and modest cellular proliferation within the middle of the stent.

The present model may be used with immunohistochemistry to investigate the molecular mechanisms associated with neointimal hyperplasia that develops in response to stent deployment and subsequent alterations in indices of wall shear stress within the stented region. In the current report, immunohistochemical analysis was used to reveal the localized expression of $\alpha$-smooth muscle actin and cellular nuclei in stented and unstented iliac arteries (Fig. 4). We plan to use the present methods to colocalize additional molecular targets using double-staining techniques. Thus, the current model may facilitate future investigation of altered fluid 
dynamics, indices of wall shear stress, and the molecular mechanisms mediating neointimal hyperplasia after stent implantation in vivo.

The current method of antegrade stent implantation may be associated with several potential limitations that warrant additional comment. Stents were relatively easily implanted in healthy rabbit iliac arteries, but technical difficulties may be encountered when stent deployment is attempted in the presence of atherosclerosis. The mechanisms responsible for neointimal hyperplasia in normal as compared with atherosclerotic vessels may also be different. Previous studies have demonstrated that the greatest inflammatory reaction to stent implantation is localized to regions where the stent is in direct apposition to the intima (Farb et al., 1999, Farb et al., 2002). Thus, the present histological results obtained in circumferentially homogenous normal rabbit iliac arteries may be different from those observed in vessels containing eccentrically localized atherosclerotic plaques. The composition of neointimal hyperplasia that develops in animals differs from that observed in humans (Bennett \& O'Sullivan, 2001). Furthermore, the onset and development of neointimal hyperplasia after stent implantation is more rapid in animals than in humans (Orford, Selwyn, Ganz, Popma, \& Rogers, 2000). Nevertheless, neointimal hyperplasia occurs consistently throughout mammalian species in response to direct stimulation of endothelial cells.

Atherosclerosis is most often precipitated by elevated concentrations of circulating low-density lipoprotein cholesterol that promote leukocyte adhesion, rolling, and extravasation (Krieglstein \& Granger, 2001). The placement of a stent may contribute to further chronic vascular injury and restenosis despite the initial restoration of blood flow distal to the atherosclerotic plaque. In the current model of neointimal hyperplasia, the initial vascular damage was instead caused by the deployment of the stent. Despite these differing mechanisms of vascular injury, the neointimal hyperplasia that occurs in animal models of stent implantation appears to closely resemble that observed in humans, albeit in an accelerated time frame (Virmani, Kolodgie, Farb, \& Lafont, 2003). Moreover, many of the current criteria required for a stent delivery device to gain Food and Drug Administration approval are based on findings from animal models of vascular injury. Thus, the current model of rabbit antegrade iliac artery stent deployment may be particularly useful for the comprehensive examination of vascular responses to stent deployment, as it provides a highly reproducible method of producing and assessing cellular proliferation after implantation (Bennett \& O'Sullivan, 2001, Narayanaswamy et al., 2000, Orford et al., 2000).

In summary, we report the details of a model of antegrade iliac artery stent placement developed to allow the investigation of the influence of stent-induced alterations in indices of wall shear stress on neointimal hyperplasia in vivo. In a preliminary investigation, neointimal hyperplasia was greatest in regions exposed to low wall shear stress and elevated spatial wall shear stress gradients (LaDisa, Warltier et al., 2004). Moreover, there was a temporally dependent inverse relationship between wall shear stress and the severity of neointimal hyperplasia. This suggests that stent implantation may introduce spatial alterations in wall shear stress that modulate the growth of neointimal hyperplasia to temporally abolish wall shear stress disparity in vivo. The present experimental methodology is highly reproducible and produces selective neointimal hyperplasia within the stented region of the vessel after implantation in the absence of cellular proliferation proximal and distal to the site of deployment. This model will facilitate additional study of the mechanisms associated with neointimal hyperplasia after stenting in the absence of complicating localized disruptions of fluid dynamics caused by retrograde implantation that may inadvertently also affect cellular proliferation.

\section{Acknowledgements}

The authors would like to thank Dorothee Weihrauch, Tarrant J. Csida, and the staff in the Center for Animal Research Imaging at the Zablocki VA Medical Center for their experimental support. This work was supported, in 
part, by National Institutes of Health grants HL-03690 (to Dr. Kersten), HL-63705 (to Dr. Kersten), HL-54820 (to Dr. Warltier), and GM-08377 (to Dr. Warltier) from the United States Public Health Service, Bethesda, MD.

\section{References}

Bennett \& O'Sullivan, 2001. M.R. Bennett, M. O'Sullivan. Mechanisms of angioplasty and stent restenosis: Implications for design of rational therapy. Pharmacology and Therapeutics, 91 (2001), pp. 149-166

Burgio et al., 1984. V.L. Burgio, A. Martini, M.A. Avanzini, M. Paulli, R. Rosso. Immunofluorescence and enzyme histochemistry on consecutive sections from glycol-methacrylate-embedded bone marrow, lymph node and kidney specimens. Applied Pathology, 2 (1984), pp. 128-134

Farb et al., 1999. A. Farb, G. Sangiorgi, A.J. Carter, V.M. Walley, W.D. Edwards, R.S. Schwartz, R. Virmani. Pathology of acute and chronic coronary stenting in humans. Circulation, 99 (1999), pp. 44-52

Farb et al., 2002. A. Farb, D.K. Weber, F.D. Kolodgie, A.P. Burke, R. Virmani. Morphological predictors of restenosis after coronary stenting in humans. Circulation, 105 (2002), pp. 2974-2980

Fischman et al., 1994. D.L. Fischman, M.B. Leon, D.S. Baim, R.A. Schatz, M.P. Savage, I. Penn, K. Detre, L. Veltri, D. Ricci, M. Nob uyoshi, M. Cleman, R. Heuser, D. Almond, P.S. Teirstein, R.D. Fish, A. Colombo, J. Brinker, J. Moses, A. Sh aknovich, J. Hirshfeld, S. Bailey, S. Ellis, R. Rake, S. Goldberg. A randomized comparison of coronarystent placement and balloon angioplasty in the treatment of coronary artery disease. Stent Restenosis Study Investigators. New England Journal of Medicine, 331 (1994), pp. 496-501

Garasic et al., 2000. J.M. Garasic, E.R. Edelman, J.C. Squire, P. Seifert, M.S. Williams, C. Rogers. Stent and artery geometry determine intimal thickening independent of arterial injury. Circulation, 101 (2000), pp. 812818

Herdeg et al., 2003. C. Herdeg, M. Fitzke, M. Oberhoff, A. Baumbach, S. Schroeder, K.R. Karsch. Effects of atorvastatin on in-stent stenosis in normo- and hypercholesterolemic rabbits. International Journal of Cardiology, 91 (2003), pp. 59-69

Holmes et al., 1998. D.R. Holmes Jr., J. Hirshfield Jr., D. Faxon, R.E. Vlietstra, A. Jacobs, S.B. King $3^{\text {rd }}$. ACC expert consensus document on coronary artery stents. Document of the American College of Cardiology. Journal of the American College of Cardiology, 32 (1998), pp. 1471-1482

Kleinstreuer et al., 2001. C. Kleinstreuer, S. Hyun, J.R. Buchanan Jr., P.W. Longest, J.P. Archie Jr., G.A. Truskey. Hemodynamic parameters and early intimal thickening in branching blood vessels. Critical Reviews in Biomedical Engineering, 29 (2001), pp. 1-64

Krieglstein \& Granger, 2001. C.F. Krieglstein, D.N. Granger. Adhesion molecules and their role in vascular disease. American Journal of Hypertension, 14 (2001), pp. 44S-54S

LaDisa et al., 2003. J.F. LaDisa Jr., I. Guler, L.E. Olson, D.A. Hettrick, J.R. Kersten, D.C. Warltier, P.S. Pagel. Threedimensional computational fluid dynamics modeling of alterations in coronary artery wall shear stress produced by stent implantation. Annals of Biomedical Engineering, 31 (2003), pp. 972-980

LaDisa et al., 2004. J.F. LaDisa, L.E. Olson, I. Guler, D.A. Hettrick, S.H. Audi, J.R. Kersten, D.C. Warltier, P.S. Pagel. Stent design properties and deployment ratio influence indices of wall shear stress: A 3D computational fluid dynamics investigation within a normal artery. Journal of Applied Physiology, 97 (2004), pp. 424-430

LaDisa et al., 2004. J.F.J. LaDisa, D.C. Warltier, L.E. Olson, S.T. Haworth, R.C. Molthen, D.A. Hettrick, et al. Distributions of wall shear stress influence neointimal hyperplasia after stent implantation in vivo. Annals of Biomedical Engineering 2004 BMES meeting in Philadelphia, PA (2004)

Liu \& Goldman, 2001. S.Q. Liu, J. Goldman. Role of blood shear stress in the regulation of vascular smooth muscle cell migration. IEEE Transactions on Biomedical Engineering, 48 (2001), pp. 474-483

Liu et al., 2002. S.Q. Liu, L. Zhong, J. Goldman. Control of the shape of a thrombus-neointima-like structure by blood shear stress. Journal of Biomechanical Engineering, 124 (2002), pp. 30-36

Narayanaswamy et al., 2000. M. Narayanaswamy, K.C. Wright, K. Kandarpa. Animal models for atherosclerosis, restenosis, and endovascular graft research. Journal of Vascular and Interventional Radiology, 11 (2000), pp. 5-17 
Orford et al., 2000. J.L. Orford, A.P. Selwyn, P. Ganz, J.J. Popma, C. Rogers. The comparative pathobiology of atherosclerosis and restenosis. American Journal of Cardiology, 86 (2000), pp. 6H-11H

Rogers \& Edelman, 1995. C. Rogers, E.R. Edelman. Endovascular stent design dictates experimental restenosis and thrombosis. Circulation, 91 (1995), pp. 2995-3001

Rogers et al., 1998. C. Rogers, E.R. Edelman, D.I. Simon. A mAb to the beta2-leukocyte integrin Mac-1 (CD11b/CD18) reduces intimal thickening after angioplasty or stent implantation in rabbits.

Proceedings of the National Academy of Sciences of the United States of America, 95 (1998), pp. 1013410139

Rogers et al., 1999. C. Rogers, D.Y. Tseng, J.C. Squire, E.R. Edelman. Balloon-artery interactions during stent placement: A finite element analysis approach to pressure, compliance, and stent design as contributors to vascular injury. Circulation Research, 84 (1999), pp. 378-383

Schwartz et al., 1992.

R.S. Schwartz, K.C. Huber, J.G. Murphy, W.D. Edwards, A.R. Camrud, R.E. Vlietstra, D.R. Holmes. Restenosis and the proportional neointimal response to coronary artery injury: Results in a porcine model. Journal of the American College of Cardiology, 19 (1992), pp. 267-274

Serruys et al., 1994. P. Serruys, P. De .

aegere, F. Kiemeneij, C. Macaya, W. Rutch, G. Heyndrickx, H. Emanuelsson, J. Marco, V. Legrand, P. Mate rne, J. Belardi, U. Sigwart, A. Colombo, J.J. Goy, P. van den Heuvel, J. Delcan, M-A. Morel. A comparison of balloon-expandible-stent implantation with balloon angioplasty in patients with coronary heart disease. New England Journal of Medicine, 331 (1994), pp. 489-495

van Beusekom et al., 1998. H.M. van Beusekom, D.M. Whelan, S.H. Hofma, S.C. Krabbendam, V.W. van Hinsbergh, P.D. Verdouw, W.J. van der Giessen. Long-term endothelial dysfunction is more pronounced after stenting than after balloon angioplasty in porcine coronary arteries. Journal of the American College of Cardiology, 32 (1998), pp. 1109-1117

Virmani et al., 2003. R. Virmani, F.D. Kolodgie, A. Farb, A. Lafont. Drug eluting stents: Are human and animal studies comparable? Heart, 89 (2003), pp. 133-138 